\title{
Article
}

\section{Oscillatory Behavior of Third-Order Quasi-Linear Neutral Differential Equations}

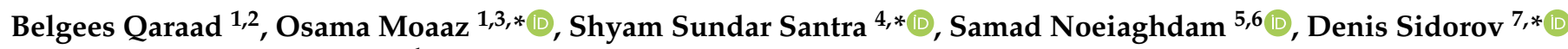 \\ and Elmetwally M. Elabbasy ${ }^{1}$
}

1 Department of Mathematics, Faculty of Science, Mansoura University, Mansoura 35516, Egypt; belgeesmath2016@students.mans.edu.eg (B.Q.); emelabbasy@mans.edu.eg (E.M.E.)

2 Department of Mathematics, Faculty of Science, Amran University, Amran 999101, Yemen

3 Section of Mathematics, International Telematic University Uninettuno, CorsoVittorio Emanuele II, 39, 00186 Roma, Italy

4 Department of Mathematics, JIS College of Engineering, Kalyani 741235, India

5 Industrial Mathematics Laboratory, Baikal School of BRICS, Irkutsk National Research Technical University, Irkutsk, 664074, Russia; snoei@istu.edu or noiagdams@susu.ru

6 Department of Applied Mathematics and Programming, South Ural State University, Lenin Prospect 76, 454080 Chelyabinsk, Russia

7 Melentiev Energy Systems Institute of Siberian Branch of Russian Academy of Science, 664033 Irkutsk, Russia;

* Correspondence: o_moaaz@mans.edu.eg (O.M.); shyam01.math@gmail.com or

shyamsundar.santra@jiscollege.ac.in (S.S.S.); sidorovdn@istu.edu or dsidorov@isem.irk.ru (D.S.)

check for

updates

Citation: Qaraad, B.; Moaaz, O.;

Santra S.S.; Noeiaghdam, S.; Sidorov,

D.; Elabbasy, E.M.; Oscillatory

Behavior of Third-Order

Quasi-Linear Neutral Differential

Equations. Axioms 2021, 10, 346.

https://doi.org/10.3390/

axioms 10040346

Academic Editor: Chris Goodrich

Received: 1 September 2021

Accepted: 10 November 2021

Published: 17 December 2021

Publisher's Note: MDPI stays neutral with regard to jurisdictional claims in published maps and institutional affiliations.

Copyright: (c) 2021 by the authors. Licensee MDPI, Basel, Switzerland. This article is an open access article distributed under the terms and conditions of the Creative Commons Attribution (CC BY) license (https:/ / creativecommons.org/licenses/by/ $4.0 /)$.

\begin{abstract}
In this paper, we consider a class of quasilinear third-order differential equations with a delay argument. We establish some conditions of such certain third-order quasi-linear neutral differential equation as oscillatory or almost oscillatory. Those criteria improve, complement and simplify a number of existing results in the literature. Some examples are given to illustrate the importance of our results.
\end{abstract}

Keywords: thrid-order differential equations; delay; oscillation criteria

\section{Introduction}

Consider the third-order neutral delay differential equation of the form

$$
\left(r(t)\left(y^{\prime \prime}(t)\right)^{\alpha}\right)^{\prime}+q(t) f(x(\sigma(t)))=0
$$

where $y(t)=x(t)+p(t) x(\tau(t))$ and we assume that the following hypotheses are satisfied: (I) $\quad r \in C\left(\left[t_{0}, \infty\right), \mathbb{R}\right)$ is positive and $\pi(t)<\infty$, where

$$
\pi(t)=\int_{t}^{\infty} r^{-1 / \alpha}(s) \mathrm{d} s
$$

(I 2$) \quad p, q \in C\left(\left[t_{0}, \infty\right), \mathbb{R}\right), p \leq p_{0}<\infty, q$ is non-negative and does not eventually vanish (i.e., $q(t)$ is not eventually zero on any half line $\left[t_{*}, \infty\right)$ for $\left.t_{*} \geq t_{0}\right)$;

(I $\left.\mathbf{I}_{3}\right) \quad \sigma, \tau \in C^{1}\left(\left[t_{0}, \infty\right), \mathbb{R}\right), \sigma(t)<t, \tau(t)<t, \tau^{\prime}(t) \geq \tau_{0}>0, \sigma \circ \tau=\tau \circ \sigma$ and $\lim _{t \rightarrow \infty} \sigma(t)=\lim _{t \rightarrow \infty} \tau(t)=\infty$;

(I) $f \in C(\mathbb{R}, \mathbb{R})$ and satisfies

$$
f(x)>k x^{\alpha} \text { for all } k>0 .
$$

where $\alpha$ is the quotient of odd positive integers.

By a solution of (1), we mean a nontrivial function $x \in C\left(\left[T_{x}, \infty\right), \mathbb{R}\right)$ with $T_{x} \geq t_{0}$, which satisfies the property $r\left(y^{\prime \prime}\right)^{\alpha} \in C^{1}\left(\left[T_{x}, \infty\right), \mathbb{R}\right)$, moreover, satisfies (1) on $\left[T_{x}, \infty\right)$. We 
only consider those solutions of (1) satisfying, on some half-line, $\left[T_{x}, \infty\right)$ and satisfying the condition $\sup \{|x(t)|: T \leq t<\infty\}>0$ for any $T \geq T_{x}$. A solution of (1) is oscillatory if it has arbitrarily large zeros on $\left[T_{x}, \infty\right)$; otherwise, it is said to be nonoscillatory. The equation itself is termed oscillatory if all its solutions oscillate, and is said to be almost oscillatory if all its solutions are oscillatory or asymptotically convergent to zero.

The neutral differential equations have numerous applications in electrical engineering, chemical reactions analysis, and economics.

Such equations are essential tools to model and study the dynamics and stability properties of electrical power systems, as in the works of Milano et al. [1,2]. The asymptotic behavior of solutions of associated delay differential equations have been used to describe the behavior of solutions to third-order partial differential equations. Additionally, they are employed for the study of distributed networks containing lossless transmission lines; see $[3,4]$ for more details.

Recently, there has been much research activity concerning the oscillation of secondorder differential equations with delay. See, for example, $[5,6]$ and the references cited therein. Compared to the development of the oscillation for the second-order equations, the oscillation for third-order equations has received considerably less attention from researchers; see [7-24].

Baculikova and Dzurina [25,26] and Grace et al. [27] considered the third-order nonlinear delay differential equation

$$
\left(r(t)\left(x^{\prime \prime}(t)\right)^{\alpha}\right)^{\prime}+q(t) f(x(\sigma(t)))=0,
$$

in the case where $\pi(t)=\infty$ or $\pi(t)<\infty$.

Saker and Dzurina [28] studied the third-order nonlinear delay differential equation

$$
\left(r(t)\left(x^{\prime \prime}(t)\right)^{\alpha}\right)^{\prime}+q(t) x^{\beta}(\sigma(t))=0,
$$

and obtained several oscillation criteria, which guarantee that all non-oscillatory solutions of such Equation (2) tend towards zero.

Ravi et al. [29] investigated a third-order delay differential equation

$$
\left(r_{2}(t)\left(r_{1}(t)\left(x^{\prime}(t)\right)\right)^{\prime}\right)^{\prime}+q(t) x^{\beta}(\sigma(t))=0,
$$

and established some oscillation results that supplemented and improved the results in [27]. Sidorov and Trufanov [30] considered nonlinear operator equations with a functional perturbation of the argument of neutral type and reduced the problem to quasilinear operator equations with a functional perturbation of the argument.

Moaaz, in [11], studied a third-order nonlinear delay differential (2) under the condition $\pi(t)=\infty$; he developed some results of previous references and established several sufficient conditions, which ensure that all solutions of (2) are oscillatory.

In previous papers, the authors used an integral averaging technique and a Riccati transformation to establish some sufficient conditions which ensure that any solution of ( 1) oscillates or converges to zero. The purpose of this paper is to improve and generalize these results and present some new sufficient conditions, which ensure that any solution of (1) oscillates or, for all its nonoscillatory solutions, tend towards zero as $t \rightarrow \infty$.

\section{Auxiliary Results}

In this section, we state and prove the following lemmas, which will be useful in the proofs of the main results. 
Lemma 1 ([29]). Assume $x(t)$ is nonoscillatory solution of (1). Then, $y(t)>0$ and there are three possible cases of $y(t)$ :

$$
\begin{array}{ll}
\mathbf{N}_{1} & y^{\prime}(t)>0, y^{\prime \prime}(t)>0, \\
\mathbf{N}_{2} & y^{\prime}(t)>0, y^{\prime \prime}(t)<0, \\
\mathbf{N}_{3} & y^{\prime}(t)<0, y^{\prime \prime}(t)>0
\end{array}
$$

Lemma 2 ([31]). Let $h(u)=A u-B(u-C)^{(\alpha+1) / \alpha}$, where $B>0, A$ and $C$ are constants, $\alpha$ be $a$ ratio of two odd positive numbers. Then, $h$ attains its maximum value on $\mathbb{R}$ at $u^{*}=C+\left(\frac{\alpha A}{(\alpha+1) B}\right)^{\alpha}$ such that

$$
\max _{u \in \mathbb{R}} h(u)=h\left(u^{*}\right)=A C+\frac{\alpha^{\alpha}}{(\alpha+1)^{\alpha+1}} \frac{A^{\alpha+1}}{B^{\alpha}} .
$$

Lemma 3 ([32]). Assume that $c_{1}, c_{2} \in[0, \infty)$ and $\gamma>0$. Then

$$
\left(c_{1}+c_{1}\right)^{\gamma} \leq \mu\left(c_{1}^{\gamma}+c_{2}^{\gamma}\right),
$$

where

$$
\mu:= \begin{cases}1 & \text { if } \gamma \leq 1 \\ 2^{\gamma-1} & \text { if } \gamma>1\end{cases}
$$

Lemma 4. Let $x$ be a positive solution of (1), $y^{\prime}(t)>0$ and $p(t) \in(0,1)$. Then,

$$
\left(r(t)\left(y^{\prime \prime}(t)\right)^{\alpha}\right)^{\prime}+k Q(t) y^{\alpha}(\sigma(t)) \leq 0
$$

where

$$
Q(t)=q(t)(1-p(\sigma(t)))^{\alpha}
$$

Proof. Assume that $x$ is a positive solution of (1). From hypothesis $\left(\mathbf{I}_{4}\right),(1)$ becomes

$$
\left(r(t)\left(y^{\prime \prime}(t)\right)^{\alpha}\right)^{\prime}+k q(t) x^{\alpha}(\sigma(t)) \leq 0
$$

Since $y^{\prime}(t)>0$, we find

$$
\begin{aligned}
x(t) & =y(t)-p(t) x(\tau(t)) \geq y(t)-p(t) y(\tau(t)) \\
& \geq y(t)-p(t) y(t)=y(t)(1-p(t)) .
\end{aligned}
$$

That is

$$
x^{\alpha}(\sigma(t)) \geq y^{\alpha}(\sigma(t))(1-p(\sigma(t)))^{\alpha} .
$$

Combining (4) and (5), we have

$$
\left(r(t)\left(y^{\prime \prime}(t)\right)^{\alpha}\right)^{\prime}+k Q(t) y^{\alpha}(\sigma(t)) \leq 0
$$

This completes the proof.

Lemma 5. Assume that $x(t)$ is a positive solution of (1). Then,

$$
\left(r(t)\left(y^{\prime \prime}(t)\right)^{\alpha}+\frac{p_{0}^{\alpha}}{\tau_{0}} r(\tau(t))\left(y^{\prime \prime}(\tau(t))\right)^{\alpha}\right)^{\prime} \leq-\frac{k}{\mu} \hat{O}(t) y^{\alpha}(\sigma(t)),
$$

where $\hat{O}(t):=\min \{q(t), q(\tau(t))\}$. 
Proof. Let $x(t)$ be a positive solution of (1). Then, there exists $t_{1} \geq t_{0}$ such that $x(\sigma(t))>0$ and $x(\tau(t))>0$ for all $t \geq t_{1}$. From Lemma 3 and $\sigma \circ \tau=\tau \circ \sigma$, we obtain

$$
y^{\alpha}(\sigma(t))=\mu\left(x^{\alpha}(\sigma(t))+p_{0}^{\alpha} x^{\alpha}(\sigma(\tau(t)))\right) .
$$

In view of $\left(\mathbf{I}_{3}\right),(4)$ implies

$$
\begin{aligned}
0 & \geq \frac{p_{0}^{\alpha}}{\tau^{\prime}(t)}\left(r(\tau(t))\left(y^{\prime \prime}(\tau(t))\right)^{\alpha}\right)^{\prime}+p_{0}^{\alpha} k q(\tau(t)) x^{\alpha}(\sigma(\tau(t))) \\
& \geq \frac{p_{0}^{\alpha}}{\tau_{0}}\left(r(\tau(t))\left(y^{\prime \prime}(\tau(t))\right)^{\alpha}\right)^{\prime}+p_{0}^{\alpha} k q(\tau(t)) x^{\alpha}(\tau(\sigma(t))) .
\end{aligned}
$$

Using (4) with the above inequality, and taking into account (7), we have

$$
\begin{aligned}
\left(r(t)\left(y^{\prime \prime}(t)\right)^{\alpha}\right)^{\prime}+\frac{p_{0}^{\alpha}}{\tau_{0}}\left(r(\tau(t))\left(y^{\prime \prime}(\tau(t))\right)^{\alpha}\right)^{\prime}+k \hat{O}(t)\left(x^{\alpha}(\sigma(t))+p_{0}^{\alpha} x^{\alpha}(\tau(\sigma(t)))\right) & \leq 0 \\
\left(r(t)\left(y^{\prime \prime}(t)\right)^{\alpha}\right)^{\prime}+\frac{p_{0}^{\alpha}}{\tau_{0}}\left(r(\tau(t))\left(y^{\prime \prime}(\tau(t))\right)^{\alpha}\right)^{\prime}+\frac{k}{\mu} \hat{O}(t) y^{\alpha}(\sigma(t)) & \leq 0 .
\end{aligned}
$$

Thus,

$$
\left(r(t)\left(y^{\prime \prime}(t)\right)^{\alpha}+\frac{p_{0}^{\alpha}}{\tau_{0}} r(\tau(t))\left(y^{\prime \prime}(\tau(t))\right)^{\alpha}\right)^{\prime}+\frac{k}{\mu} \hat{O}(t) y^{\alpha}(\sigma(t)) \leq 0 .
$$

This completes the proof.

Lemma 6. Assume that $x(t)$ is a positive solution of (1) and $y^{\prime}(t)>0$. Then

$$
y(\sigma(t)) \geq \dot{c} \sigma(t), \text { where } c:=c \varsigma .
$$

Proof. Since $y^{\prime}$ is nondecreasing, this implies that

$$
y^{\prime}(t) \geq y^{\prime}\left(t_{1}\right)=: c \text { on }\left[t_{1}, \infty\right) .
$$

Integrating from $\sigma(t)$ to $t_{1}$, we get

$$
y(\sigma(t)) \geq c\left(\sigma(t)-t_{1}\right) .
$$

Hence, for any $\varsigma \in(0,1)$ and $t \geq t_{2}$, we see that

$$
y(\sigma(t)) \geq \dot{c} \sigma(t) .
$$

The proof is complete.

Lemma 7. Let $x(t)$ be a positive solution of (1). If

$$
\int_{t_{0}}^{\infty} \hat{O}(t) \sigma^{\alpha}(t) \mathrm{d} t=\infty,
$$

then case $\mathbf{N}_{1}$ is impossible. 
Proof. Assume that $x(t)$ is a positive solution of (1) on $\left[t_{0}, \infty\right)$. Then, there exists $t_{1} \geq t_{0}$ such that $x(\tau(t))>0$ and $x(\sigma(t))>0$ for all $t \geq t_{1}$. On the contrary, assume that $y(t)$ satisfies case $\mathbf{N}_{1}$. Integrating (6) from $t_{2}$ to $t$ and using (8), we get

$$
\begin{aligned}
& r(t)\left(y^{\prime \prime}(t)\right)^{\alpha}+\frac{p_{0}^{\alpha}}{\tau_{0}} r(\tau(t))\left(y^{\prime \prime}(\tau(t))\right)^{\alpha} \\
\leq & r\left(t_{2}\right)\left(y^{\prime \prime}\left(t_{2}\right)\right)^{\alpha}+\frac{p_{0}^{\alpha}}{\tau_{0}} r\left(\tau\left(t_{2}\right)\right)\left(y^{\prime \prime}\left(\tau\left(t_{2}\right)\right)\right)^{\alpha}-\left(c^{\prime}\right)^{\alpha} \frac{k}{\mu} \int_{t_{2}}^{t} \hat{O}(t) \sigma^{\alpha}(t) \mathrm{d} t,
\end{aligned}
$$

which is a contradiction.

Lemma 8. Let $y(t)$ be a positive increasing solution of (1). If

$$
\int_{t_{0}}^{\infty} \frac{1}{r^{1 / \alpha}(t)}\left(\int_{t_{0}}^{t} \hat{O}(t) \sigma^{\alpha}(s) \mathrm{d} s\right)^{1 / \alpha} \mathrm{d} t=\infty,
$$

then $y$ satisfies case $\mathbf{N}_{2}$ for $t \geq t_{1}$ and

(a) $y(t) \geq t y^{\prime}(t)$ and $y(t) / t$ is decreasing, and $\lim _{t \rightarrow \infty} y(t) / t=y^{\prime}=0$,

(b) $y^{\prime}(t) \geq-\pi(t) r^{\frac{1}{\alpha}}(t) y^{\prime \prime}(t)$ and $y^{\prime}(t) / \pi(t)$ is increasing.

Proof. Assume that $x(t)$ is a positive solution of (1) on $\left[t_{0}, \infty\right)$. Then, there exists $t_{1} \geq t_{0}$ such that $x(\tau(t))>0$ and $x(\sigma(t))>0$ for all $t \geq t_{1}$. Since $y$ is increasing, $y$ satisfies either case $\mathbf{N}_{1}$ or $\mathbf{N}_{2}$. In view of $\pi(t)<\infty$ and (11), we see that (9) hold. By Lemma 7, $y(t)$ satisfies case $\mathbf{N}_{2}$.

On the other hand, it follows from $y^{\prime}(t)$ is decreasing, such that there exists a constant $\lambda \geq 0$ such that $\lim _{t \rightarrow \infty} y^{\prime}(t)=\lambda \geq 0$. We claim that $\lambda=0$. As the proof of Lemma 7 we have (10). Take into account $\left(r(t)\left(y^{\prime \prime}(t)\right)^{\alpha}\right)^{\prime} \leq 0$ and $y^{\prime \prime}(t)<0$, we have

$$
r(t)\left(y^{\prime \prime}(t)\right)^{\alpha}\left(1+\frac{p_{0}^{\alpha}}{\tau_{0}}\right) \leq-(\hat{c})^{\alpha} \frac{k}{\mu} \int_{t_{1}}^{t} \hat{O}(s) \sigma^{\alpha}(s) \mathrm{d} s .
$$

It follows that

$$
y^{\prime \prime}(t) \leq-\hat{c}\left(\frac{1}{r(t)}\left(\frac{k \tau_{0}}{\mu\left(\tau_{0}+p_{0}^{\alpha}\right)}\right)\right)^{1 / \alpha}\left(\int_{t_{1}}^{t} \hat{O}(s) \sigma^{\alpha}(s) \mathrm{d} s\right)^{1 / \alpha} .
$$

Integrating from $t_{2}$ to $t$, we obtain

$$
y^{\prime}(t) \leq y^{\prime}\left(t_{2}\right)-\dot{c}\left(\frac{k \tau_{0}}{\mu\left(\tau_{0}+p_{0}^{\alpha}\right)}\right)^{\frac{1}{\alpha}} \int_{t_{2}}^{t} \frac{\left(\int_{t_{2}}^{u} \hat{O}(s) \sigma^{\alpha}(s) \mathrm{d} s\right)^{\frac{1}{\alpha}}}{r^{\frac{1}{\alpha}}(u)} \mathrm{d} u .
$$

In view of (11), this contradicts the positivity of $y^{\prime}(t)$. Thus $\lambda=0$. By "Hospital's rule", we see that

$$
\lim _{t \rightarrow \infty} \frac{y(t)}{t}=0 \text { and } \lim _{t \rightarrow \infty} y^{\prime}(t)=0 .
$$

Thus,

$$
y\left(t_{1}\right)-y^{\prime}(t) t_{1}>0
$$

Therefore,

$$
y(t)>y^{\prime}(t) t_{1}
$$

for $t \geq t_{2}$. Hence, by the monotonicity of $y^{\prime}(t)$, one can obtain that

$$
y(t)=y\left(t_{1}\right)+\int_{t_{1}}^{t} y^{\prime}(s) \mathrm{d} s \geq y\left(t_{1}\right)+y^{\prime}(t)\left(t-t_{1}\right) .
$$


By (12), we have

$$
\left(\frac{y(t)}{t}\right)^{\prime}=\frac{t y^{\prime}-y}{t^{2}}<0
$$

Now, it is easy to see that

$$
y^{\prime}(t) \geq-\int_{t}^{\infty} \frac{1}{r^{1 / \alpha}(s)} r^{1 / \alpha}(s) y^{\prime \prime}(s) \mathrm{d} s \geq-r^{1 / \alpha}(t) y^{\prime \prime}(t) \pi(t)
$$

Thus,

$$
\left(\frac{y^{\prime}(t)}{\pi(t)}\right)^{\prime}=\frac{r^{1 / \alpha}(t) y^{\prime \prime}(t) \pi(t)+y^{\prime}(t)}{r^{1 / \alpha}(t) \pi^{2}(t)} \geq 0 .
$$

The proof is complete.

\section{Main Results}

Theorem 1. If

$$
\int_{t_{0}}^{\infty} \frac{1}{r^{1 / \alpha}(t)}\left(\int_{t_{0}}^{t} \hat{O}(s) \mathrm{d} s\right)^{1 / \alpha} \mathrm{d} t=\infty,
$$

then possible positive solution of (1) satisfies case $\mathbf{N}_{3}$.

Proof. Assume that $x(t)$ is a positive solution of (1) on $\left[t_{0}, \infty\right)$. Then, there exists $t_{1} \geq t_{0}$ such that $x(\tau(t))>0$ and $x(\sigma(t))>0$ for all $t \geq t_{1}$. Suppose that $y(t)$ satisfies case $\mathbf{N}_{1}$ or $\mathbf{N}_{2}$. Since $y$ is increasing, then it follows that

$$
y(t) \geq y\left(t_{1}\right)=\varrho \text { for } t \geq t_{2}
$$

Set

$$
\omega(t)=r(t)\left(y^{\prime \prime}(t)\right)^{\alpha}+\frac{p_{0}^{\alpha}}{\tau_{0}} r(\tau(t))\left(y^{\prime \prime}(\tau(t))\right)^{\alpha} .
$$

In (6), we obtain

$$
\omega^{\prime}(t) \leq-\frac{k}{\mu} \hat{O}(t) y^{\alpha}(\sigma(t))
$$

Since $\omega^{\prime}(t) \leq 0$, by (15), we have

$$
\omega(t) \geq r(t)\left(y^{\prime \prime}(t)\right)^{\alpha}\left(1+\frac{p_{0}^{\alpha}}{\tau_{0}}\right)
$$

Integrating (16) from $t_{2}$ to $t$ and using (14), we obtain

$$
\omega(t) \leq \omega\left(t_{1}\right)-\frac{\varrho^{\alpha} k}{\mu} \int_{t_{2}}^{t} \hat{O}(s) \mathrm{d} s .
$$

First, let $y(t)$ satisfies case $\mathbf{N}_{1}$. We note that $\omega(t)>0$. Using the fact $\pi(t)<\infty$ together with (13) yields that $\int_{t_{0}}^{t} \hat{O}(s)$ ds contradicts the positivity of $\omega(t)$.

If $y(t)$ satisfies case $\mathbf{N}_{2}$, using (17) in (18) becomes

$$
r(t)\left(y^{\prime \prime}(t)\right)^{\alpha}\left(1+\frac{p_{0}^{\alpha}}{\tau_{0}}\right) \leq-\frac{\varrho^{\alpha} k}{\mu} \int_{t_{2}}^{t} \hat{O}(s) \mathrm{d} s,
$$

that is

$$
y^{\prime \prime}(t) \leq-\varrho\left(\frac{k \tau_{0}}{\mu\left(\tau_{0}+p_{0}^{\alpha}\right)}\right)^{\frac{1}{\alpha}}\left(\frac{1}{r(t)} \int_{t_{2}}^{t} \hat{O}(s) \mathrm{d} s\right)^{\frac{1}{\alpha}}
$$


Integrating from $t_{2}$ to $t$, we have

$$
y^{\prime}(t) \leq y^{\prime}\left(t_{2}\right)-\varrho\left(\frac{k \tau_{0}}{\mu\left(\tau_{0}+p_{0}^{\alpha}\right)}\right)^{\frac{1}{\alpha}} \int_{t_{2}}^{t}\left(\frac{1}{r(u)} \int_{t_{2}}^{u} \hat{O}(s) \mathrm{d} s\right)^{\frac{1}{\alpha}} \mathrm{d} u .
$$

we obtain a cotradiction with the positivity of $y^{\prime}(t)$. The proof of the theorem is complete.

Theorem 2. If

$$
\liminf _{t \rightarrow \infty} \int_{\sigma(t)}^{t}\left(\frac{1}{r(u)} \int_{t_{2}}^{u} \hat{O}(s) \sigma^{\alpha}(s) \mathrm{d} s\right)^{\frac{1}{\alpha}} \mathrm{d} u>\left(\frac{\mu\left(\tau_{0}+p_{0}^{\alpha}\right)}{k \tau_{0} e^{\alpha}}\right)^{\frac{1}{\alpha}},
$$

then, a possible positive solution to (1) satisfies case $\mathbf{N}_{3}$.

Proof. Assume that $x(t)$ is a positive solution of (1) on $\left[t_{0}, \infty\right)$. Then, there exists $t_{1} \geq t_{0}$ such that $x(\tau(t))>0$ and $x(\sigma(t))>0$ for all $t \geq t_{1}$. Suppose that $y$ satisfies case $\mathbf{N}_{1}$ or $\mathbf{N}_{2}$. In view of (19), (11) holds. Hence, by Lemma $8, y(t)$ satisfies case $\mathbf{N}_{2}$ and properties (a) and (b) in Lemma 8. This implies that

$$
y(\sigma(t)) \geq \sigma(t) y^{\prime}(\sigma(t)) .
$$

Combining the above inequality along with (6), we get

$$
\left(r\left(y^{\prime \prime}(t)\right)^{\alpha}+\frac{p_{0}^{\alpha}}{\tau_{0}} r\left(y^{\prime \prime}(\tau(t))\right)^{\alpha}\right)^{\prime} \leq-\frac{k}{\mu} \hat{O}(t) \sigma^{\alpha}(t)\left(y^{\prime}(\sigma(t))\right)^{\alpha} .
$$

Integrating from $t_{2}$ to $t$ and using (17), we have

$$
-r(t)\left(y^{\prime \prime}(t)\right)^{\alpha}\left(1+\frac{p_{0}^{\alpha}}{\tau_{0}}\right) \geq \frac{k}{\mu} \int_{t_{2}}^{t} \hat{O}(s) \sigma^{\alpha}(s)\left(y^{\prime}(\sigma(t))\right)^{\alpha} \mathrm{d} s .
$$

Using the fact that $y^{\prime \prime}(t)<0$, we see that

$$
-y^{\prime \prime}(t) \geq\left(\frac{k \tau_{0}}{\mu\left(\tau_{0}+p_{0}^{\alpha}\right)}\right)^{\frac{1}{\alpha}} y^{\prime}(\sigma(t))\left(\frac{1}{r(t)} \int_{t_{2}}^{t} \hat{O}(s) \sigma^{\alpha}(s) \mathrm{d} s\right)^{\frac{1}{\alpha}} .
$$

Now, set $\chi(t)=y^{\prime}(t)$; we obtain

$$
\chi^{\prime}(t)+\left(\frac{k \tau_{0}}{\mu\left(\tau_{0}+p_{0}^{\alpha}\right)}\right)^{\frac{1}{\alpha}}\left(\frac{1}{r(t)} \int_{t_{2}}^{t} \hat{O}(s) \sigma^{\alpha}(s) \mathrm{d} s\right)^{\frac{1}{\alpha}} \chi(\sigma(t)) \leq 0 .
$$

In view of ([13], Theorem 1), however, the associated delay Equation (21) has a positive solution, which is a contradiction. The proof is complete.

Remark 1. Theorem 2 does not require the existence of auxiliary functions such as ([27], Theorem3), which uses the same principles (compared with first-order delay equations).

Theorem 3. Assume that (11) hold. If

$$
\limsup _{t \rightarrow \infty} \pi^{\alpha}(t) \int_{t_{0}}^{t} \hat{O}(s) \sigma^{\alpha}(s) \mathrm{d} s>\frac{\mu\left(\tau_{0}+p_{0}^{\alpha}\right)}{k \tau_{0}},
$$

then, the possible positive solution to (1) satisfies case $\mathbf{N}_{3}$. 
Proof. Suppose that $y$ satisfies case $\mathbf{N}_{1}$ or $\mathbf{N}_{2}$. We see that (9) holds due to $\pi(t)<\infty$ (this mean that $\lim _{t \rightarrow \infty} \pi(t)=0$ ) and condition (22). Hence, by Lemma $8, y(t)$ satisfies case $\mathbf{N}_{2}$ in addition to properties (a) and (b) in Lemma 8. As in the proof of Theorem 2 with the fact $r(t)\left(y^{\prime \prime}(t)\right)^{\alpha}$ is nonincreasing, and from (20), we obtain

$$
-r(t)\left(y^{\prime \prime}(t)\right)^{\alpha}\left(1+\frac{p_{0}^{\alpha}}{\tau_{0}}\right) \geq-\frac{k}{\mu} \pi^{\alpha}(t) r(t)\left(y^{\prime \prime}(t)\right)^{\alpha} \int_{t_{2}}^{t} \hat{O}(s) \sigma^{\alpha}(s) \mathrm{d} s .
$$

That is,

$$
\frac{k \tau_{0}}{\mu\left(\tau_{0}+p_{0}^{\alpha}\right)} \pi^{\alpha}(t) \int_{t_{2}}^{t} \hat{O}(s) \sigma^{\alpha}(s) \mathrm{d} s \leq 1 .
$$

This contradicts (22). The proof is complete.

Theorem 4. Assume that (11) holds. If $\sigma^{\prime}(t)>0$ and there exists a nondecreasing function $\rho \in C^{1}\left(\left[t_{0}, \infty\right),(0, \infty)\right)$, such that

$$
\limsup _{t \rightarrow \infty} \int_{T}^{t}\left(\left(\frac{k \tau_{0}}{\mu\left(\tau_{0}+p_{0}^{\alpha}\right)}\right)^{\frac{1}{\alpha}} \frac{\rho(u)}{\sigma(u) r^{\frac{1}{\alpha}}(u)} \int_{t_{0}}^{t} \hat{O}(s) \sigma^{\alpha}(s) \mathrm{d} s-\frac{\rho^{\prime 2}(u)}{4 \rho(u) \sigma^{\prime}(u)}\right) \mathrm{d} u=\infty,
$$

for any $T \in\left[t_{0}, \infty\right)$, then a possible positive solution to (1) satisfies case $\mathbf{N}_{3}$.

Proof. Assume that $x(t)$ is a positive solution of (1) on $\left[t_{0}, \infty\right)$. Then, there exists $t_{1} \geq t_{0}$ such that $x(\tau(t))>0$ and $x(\sigma(t))>0$ for all $t \geq t_{1}$. Suppose that $y$ satisfies case $\mathbf{N}_{1}$ or $\mathbf{N}_{2}$. By Lemma $8, y(t)$ satisfies case $\mathbf{N}_{2}$ and the properties (a) and (b). Define the function $w(t)$ by

$$
w(t):=\rho(t) \frac{y^{\prime}(t)}{y(\sigma(t))} .
$$

Then $w(t)>0$, and

$$
w^{\prime}(t)=\frac{\rho^{\prime}(t)}{\rho(t)} w(t)+\frac{\rho(t) y^{\prime \prime}(t)}{y(\sigma(t))}-\frac{\rho(t) y^{\prime}(t) y^{\prime}(\sigma(t)) \sigma^{\prime}(t)}{y^{2}(\sigma(t))} .
$$

Using the fact that $y^{\prime}(t)$ is decreasing, we have

$$
\begin{aligned}
w^{\prime}(t) & \leq \frac{\rho^{\prime}(t) w(t)}{\rho(t)}+\rho(t) \frac{y^{\prime \prime}(t)}{y(\sigma(t))}-\rho(t) \frac{\left(y^{\prime}(t)\right)^{2} \sigma^{\prime}(t)}{y^{2}(\sigma(t))} \\
& =\frac{\rho^{\prime}(t) w(t)}{\rho(t)}+\rho(t) \frac{y^{\prime \prime}(t)}{y(\sigma(t))}-\frac{\sigma^{\prime}(t)}{\rho(t)}\left(\rho(t) \frac{y^{\prime}(t)}{y(\sigma(t))}\right)^{2} .
\end{aligned}
$$

By (24), we obtain

$$
w^{\prime}(t) \leq \frac{\rho^{\prime}(t) w(t)}{\rho(t)}+\rho(t) \frac{y^{\prime \prime}(t)}{y(\sigma(t))}-\frac{\sigma^{\prime}(t)}{\rho(t)} w^{2}(t) .
$$

Integrating (6) from $t_{2}$ to $t$ and $(y(\sigma(t)) / \sigma(t))^{\prime}<0$, we have 


$$
\begin{aligned}
-\left(r(t)\left(y^{\prime \prime}(t)\right)^{\alpha}+\frac{p_{0}^{\alpha}}{\tau_{0}} r(\tau(t))\left(y^{\prime \prime}(\tau(t))\right)^{\alpha}\right) \geq & -\left(r\left(t_{2}\right)\left(y^{\prime \prime}\left(t_{2}\right)\right)^{\alpha}+\frac{p_{0}^{\alpha}}{\tau_{0}} r\left(t_{2}\right)\left(y^{\prime \prime}\left(\tau\left(t_{2}\right)\right)\right)^{\alpha}\right) \\
& +\frac{k}{\mu} \int_{t_{2}}^{t} \hat{O}(s) y^{\alpha}(\sigma(s)) \mathrm{d} s \\
\geq & -\left(r\left(t_{2}\right)\left(y^{\prime \prime}\left(t_{2}\right)\right)^{\alpha}+\frac{p_{0}^{\alpha}}{\tau_{0}} r\left(t_{2}\right)\left(y^{\prime \prime}\left(\tau\left(t_{2}\right)\right)\right)^{\alpha}\right) \\
& +\frac{k}{\mu}\left(\frac{y(\sigma(t))}{\sigma(t)}\right)^{\alpha} \int_{t_{2}}^{t} \hat{O}(s) \sigma^{\alpha}(s) \mathrm{d} s .
\end{aligned}
$$

Since $\lim _{t \rightarrow \infty} y(t) / t=0$, there exists $t_{3}>t_{2}$ such that

$$
-\left(r\left(t_{2}\right)\left(y^{\prime \prime}\left(t_{2}\right)\right)^{\alpha}+\frac{p_{0}^{\alpha}}{\tau_{0}} r\left(t_{2}\right)\left(y^{\prime \prime}\left(\tau\left(t_{2}\right)\right)\right)^{\alpha}\right)-\frac{k}{\mu}\left(\frac{y(\sigma(t))}{\sigma(t)}\right)^{\alpha} \int_{t_{0}}^{t_{2}} \hat{O}(s) \sigma^{\alpha}(s) \mathrm{d} s>0 .
$$

Combining the above inequality in (27) implies

$$
\begin{aligned}
-\left(r(t)\left(y^{\prime \prime}(t)\right)^{\alpha}+\frac{p_{0}^{\alpha}}{\tau_{0}} r(\tau(t))\left(y^{\prime \prime}(\tau(t))\right)^{\alpha}\right) \geq & -\left(r\left(y^{\prime \prime}\left(t_{2}\right)\right)^{\alpha}+\frac{p_{0}^{\alpha}}{\tau_{0}} r\left(y^{\prime \prime}\left(\tau\left(t_{2}\right)\right)\right)\right)^{\alpha} \\
& +\frac{k}{\mu}\left(\frac{y(\sigma(t))}{\sigma(t)}\right)^{\alpha} \int_{t_{0}}^{t} \hat{O}(s) \sigma^{\alpha}(s) \mathrm{d} s \\
& -\frac{k}{\mu}\left(\frac{y(\sigma(t))}{\sigma(t)}\right)^{\alpha} \int_{t_{0}}^{t_{2}} \hat{O}(s) \sigma^{\alpha}(s) \mathrm{d} s \\
& \geq \frac{k}{\mu}\left(\frac{y(\sigma(t))}{\sigma(t)}\right)^{\alpha} \int_{t_{0}}^{t} \hat{O}(s) \sigma^{\alpha}(s) \mathrm{d} s .
\end{aligned}
$$

Using (17), we have

$$
-r(t)\left(y^{\prime \prime}(t)\right)^{\alpha}\left(1+\frac{p_{0}^{\alpha}}{\tau_{0}}\right) \geq \frac{k}{\mu}\left(\frac{y(\sigma(t))}{\sigma(t)}\right)^{\alpha} \int_{t_{0}}^{t} \hat{O}(s) \sigma^{\alpha}(s) \mathrm{d} s,
$$

that is,

$$
\frac{y^{\prime \prime}(t)}{y(\sigma(t))} \leq-\left(\frac{k \tau_{0}}{\mu\left(\tau_{0}+p_{0}^{\alpha}\right)}\right)^{\frac{1}{\alpha}} \frac{1}{\sigma(t) r^{\frac{1}{\alpha}}(t)} \int_{t_{0}}^{t} \hat{O}(s) \sigma^{\alpha}(s) \mathrm{d} s .
$$

Substituting (27) in (25), yields

$$
\begin{aligned}
w^{\prime}(t) \leq & \frac{\rho^{\prime}(t)}{\rho(t)} w(t)-\frac{\rho(t)}{\sigma(s)}\left(\frac{k \tau_{0}}{\mu\left(\tau_{0}+p_{0}^{\alpha}\right)}\right)^{\frac{1}{\alpha}} \frac{1}{r^{\frac{1}{\alpha}}(t)} \int_{t_{0}}^{t} \hat{O}(s) \sigma^{\alpha}(s) \mathrm{d} s-\frac{\sigma^{\prime}(t)}{\rho(t)} w^{2}(t) \\
= & -\frac{\sigma^{\prime}(t)}{\rho(t)}\left(w(t)-\frac{\rho^{\prime}(t)}{2 \sigma^{\prime}(t)}\right)^{2}+\frac{\rho^{\prime 2}(t)}{4 p(t) \sigma^{\prime}(t)} \\
& -\frac{\rho(t)}{\sigma(s)}\left(\frac{k \tau_{0}}{\mu\left(\tau_{0}+p_{0}^{\alpha}\right)}\right)^{\frac{1}{\alpha}} \frac{1}{r^{\frac{1}{\alpha}}(t)} \int_{t_{0}}^{t} \hat{O}(s) \sigma^{\alpha}(s) \mathrm{d} s .
\end{aligned}
$$

Hence,

$$
w^{\prime}(t) \leq-\frac{\rho(t)}{\sigma(s)}\left(\frac{k \tau_{0}}{\mu\left(\tau_{0}+p_{0}^{\alpha}\right)}\right)^{\frac{1}{\alpha}} \frac{1}{r^{\frac{1}{\alpha}}(t)} \int_{t_{0}}^{t} \hat{O}(s) \sigma^{\alpha}(s) \mathrm{d} s+\frac{\rho^{\prime 2}(t)}{4 p(t) \sigma^{\prime}(t)} .
$$


Integrating from $t_{3}$ to $t$, we have

$$
w(t) \leq w\left(t_{3}\right)-\int_{t_{3}}^{t}\left(\left(\frac{k \tau_{0}}{\mu\left(\tau_{0}+p_{0}^{\alpha}\right)}\right)^{\frac{1}{\alpha}} \frac{\rho(u)}{\sigma(u) r^{\frac{1}{\alpha}}(u)} \int_{t_{0}}^{t} \hat{O}(s) \sigma^{\alpha}(s) \mathrm{d} s-\frac{\rho^{\prime 2}(u)}{4 p(u) \sigma^{\prime}(u)}\right) \mathrm{d} u,
$$

which is a contradiction. The proof is complete.

By choosing $\rho(t)=\frac{1}{\pi}$, we conclude the following corollary

Corollary 1. Assume that (11) holds. If there is a nondecreasing function $\rho \in C^{1}\left(\left[t_{0}, \infty\right),(0, \infty)\right)$ and $\sigma^{\prime}(t)>0$, such that

$$
\limsup _{t \rightarrow \infty} \int_{T}^{t}\left(\frac{\left(\frac{k \tau_{0}}{\mu\left(\tau_{0}+p_{0}^{\alpha}\right)}\right)^{\frac{1}{\alpha}}}{\pi(u) \sigma(u) r^{\frac{1}{\alpha}}(u)} \int_{t_{0}}^{t} \hat{O}(s) \sigma^{\alpha}(s) \mathrm{d} s-\frac{r^{-2 / \alpha}(u)}{4 \sigma^{\prime}(u) \pi^{3}(u)}\right) \mathrm{d} u=\infty,
$$

for any $T \in\left[t_{0}, \infty\right)$, then possible positive solution of (1) satisfies case $\mathbf{N}_{3}$.

Theorem 5. Assume that (11) holds. If there is a nondecreasing function $\delta \in C^{1}\left(\left[t_{0}, \infty\right),(0, \infty)\right)$, such that

$$
\limsup _{t \rightarrow \infty} \int_{t_{2}}^{t}\left(\delta(s) k \frac{\hat{O}(s)}{\mu}-\frac{\left(\delta^{\prime}(s)\right)^{\alpha+1}}{(\alpha+1)^{\alpha+1}\left(\sigma^{\prime}(s)\right)^{\alpha} \pi^{\alpha}(s) \delta^{\alpha}(s)}\right) \mathrm{d} s>\frac{\delta(t)}{\pi^{\alpha}(t) \sigma^{\alpha}(t)},
$$

then, the possible positive solution to (1) satisfies case $\mathbf{N}_{3}$.

Proof. Assume that $x(t)$ is a positive solution of (1) on $\left[t_{0}, \infty\right)$; then, there exists $t_{1} \geq t_{0}$ such that $x(\tau(t))>0$ and $x(\sigma(t))>0$ for all $t \geq t_{1}$. Suppose that $y$ satisfies case $\mathbf{N}_{1}$ or $\mathbf{N}_{2}$. By Lemma $8, y(t)$ satisfies case $\mathbf{N}_{2}$ and the properties (a) and (b).

Define the function $w(t)$ by

$$
w(t):=\delta(t)\left(\frac{r\left(y^{\prime \prime}(t)\right)^{\alpha}}{y^{\alpha}(\sigma(t))}+\frac{1}{\pi^{\alpha} \sigma^{\alpha}(t)}\right) .
$$

From Lemma 8, it is easy to see that

$$
y(\sigma(t)) \geq \sigma(t) y^{\prime}(\sigma(t)) \geq \sigma(t) y^{\prime}(t) \geq-\sigma(t) \pi(t) r^{\frac{1}{\alpha}}(t) y^{\prime \prime}(t) .
$$

That is, $w(t)>0$ and

$$
-\frac{\delta(t) r\left(y^{\prime \prime}(t)\right)^{\alpha}}{y^{\alpha}(\sigma(t))} \leq \frac{\delta(t)}{\pi^{\alpha} \sigma^{\alpha}(t)} .
$$

Using (16) and the fact $y^{\prime}(t)$ is decreasing, we have. 


$$
\begin{aligned}
w^{\prime}(t)= & \frac{\delta^{\prime}(t)}{\delta(t)} w(t)+\frac{\delta(t)\left(r(t)\left(y^{\prime \prime}(t)\right)^{\alpha}\right)^{\prime}}{y^{\alpha}(\sigma(t))}-\frac{\alpha \delta(t) r(t)\left(y^{\prime \prime}(t)\right)^{\alpha} y^{\prime}(\sigma(t)) \sigma^{\prime}(t)}{y^{\alpha+1}(\sigma(t))} \\
& +\frac{\alpha \delta(t)}{(\pi(t) \sigma(t))^{\alpha+1}}\left(\frac{\sigma(t)}{r^{\frac{1}{\alpha}}(t)}-\sigma^{\prime}(t) \pi(t)\right) \\
\leq & \frac{\delta^{\prime}(t)}{\delta(t)} w(t)-\delta(t) \frac{k}{\mu} \hat{O}(t) \\
& -\frac{\alpha \sigma^{\prime}(t) y^{\prime}(t)}{\delta^{\frac{1}{\alpha}}(t) r^{\frac{1}{\alpha}}(t) y^{\prime \prime}(t)}\left(w(t)-\frac{\delta(t)}{\pi(t) \sigma(t)}\right)^{\frac{1}{\alpha}+1}+\frac{\alpha \delta(t)}{(\pi(t) \sigma(t))^{\alpha+1}}\left(\frac{\sigma(t)}{r^{\frac{1}{\alpha}}(t)}-\sigma^{\prime}(t) \pi(t)\right) .
\end{aligned}
$$

In view of (b) in Lemma 8, we find

$$
\begin{aligned}
w^{\prime}(t) \leq & \frac{\delta^{\prime}(t)}{\delta(t)} w(t)-\delta(t) k \frac{\hat{O}(t)}{\mu} \\
& -\frac{\alpha \sigma^{\prime}(t) \pi(t)}{\delta^{\frac{1}{\alpha}}(t)}\left(w(t)-\frac{\delta(t)}{\pi^{\alpha}(t) \sigma^{\alpha}(t)}\right)^{\frac{1}{\alpha}+1}+\frac{\alpha \delta(t)}{(\pi(t) \sigma(t))^{\alpha+1}}\left(\frac{\sigma(t)}{r^{\frac{1}{\alpha}}(t)}-\sigma^{\prime}(t) \pi(t)\right) .
\end{aligned}
$$

Set

$$
A:=\frac{\delta^{\prime}(t)}{\delta(t)}, \quad B:=\frac{\alpha \sigma^{\prime}(t) \pi(t)}{\delta^{\frac{1}{\alpha}}(t)}, C:=\frac{\delta(t)}{\pi^{\alpha}(t) \sigma^{\alpha}(t)} .
$$

Using Lemma 2, we obtain

$$
\begin{aligned}
w^{\prime}(t)= & -\delta(t) k \frac{\hat{O}(t)}{\mu}+\frac{\delta^{\prime}(t)}{\pi^{\alpha}(t) \sigma^{\alpha}(t)}+\frac{1}{(\alpha+1)^{\alpha+1}} \frac{\left(\delta^{\prime}(t)\right)^{\alpha+1}}{\left(\sigma^{\prime}(t)\right)^{\alpha} \pi^{\alpha}(t) \delta^{\alpha}(t)} \\
& +\frac{\alpha \delta(t)}{(\pi(t) \sigma(t))^{\alpha+1}}\left(\frac{\sigma(t)}{r^{\frac{1}{\alpha}}(t)}-\sigma^{\prime}(t) \pi(t)\right) .
\end{aligned}
$$

It is clear that

$$
\left(\frac{\delta(t)}{\pi^{\alpha}(t) \sigma^{\alpha}(t)}\right)^{\prime}=\frac{\delta^{\prime}(t)}{\pi^{\alpha}(t) \sigma^{\alpha}(t)}+\frac{\alpha \delta(t)}{(\pi(t) \sigma(t))^{\alpha+1}}\left(\frac{\sigma(t)}{r^{\frac{1}{\alpha}}(t)}-\sigma^{\prime}(t) \pi(t)\right) .
$$

In (33), we obtain

$$
w^{\prime}(t)=-\delta(t) k \frac{\hat{O}(t)}{\mu}+\left(\frac{\delta(t)}{\pi^{\alpha}(t) \sigma^{\alpha}(t)}\right)^{\prime}+\frac{1}{(\alpha+1)^{\alpha+1}} \frac{\left(\delta^{\prime}(t)\right)^{\alpha+1}}{\left(\sigma^{\prime}(t)\right)^{\alpha} \pi^{\alpha}(t) \delta^{\alpha}(t)} .
$$

Integrating the above inequality from $t_{2}$ to $t$ yields

$$
\begin{aligned}
& \int_{t_{2}}^{t}\left(\delta(s) k \frac{\hat{O}(s)}{\mu}-\frac{\left(\delta^{\prime}(s)\right)^{\alpha+1}}{(\alpha+1)^{\alpha+1}\left(\sigma^{\prime}(s)\right)^{\alpha} \pi^{\alpha}(s) \delta^{\alpha}(s)}\right) \mathrm{d} s+\frac{\delta(t)}{\pi^{\alpha}(t) \sigma^{\alpha}(t)}-\frac{\delta\left(t_{2}\right)}{\pi^{\alpha}\left(t_{2}\right) \sigma^{\alpha}\left(t_{2}\right)} \\
\leq & w\left(t_{2}\right)-w(t) .
\end{aligned}
$$


From (30), we are led to

$$
\begin{aligned}
& \int_{t_{2}}^{t}\left(\delta(s) k \frac{\hat{O}(s)}{\mu}-\frac{\left(\delta^{\prime}(s)\right)^{\alpha+1}}{(\alpha+1)^{\alpha+1}\left(\sigma^{\prime}(s)\right)^{\alpha} \pi^{\alpha}(s) \delta^{\alpha}(s)}\right) \mathrm{d} s+\frac{\delta(t)}{\pi^{\alpha}(t) \sigma^{\alpha}(t)} \\
& -\frac{\delta\left(t_{2}\right)}{\pi^{\alpha}\left(t_{2}\right) \sigma^{\alpha}\left(t_{2}\right)} \\
\leq & \delta\left(t_{2}\right)\left(\frac{r\left(y^{\prime \prime}\left(t_{2}\right)\right)^{\alpha}}{y^{\alpha}\left(\sigma\left(t_{2}\right)\right)}\right)-\delta(t)\left(\frac{r\left(y^{\prime \prime}(t)\right)^{\alpha}}{y^{\alpha}(\sigma(t))}\right) .
\end{aligned}
$$

By (32), (35) becomes

$$
\int_{t_{2}}^{t}\left(\delta(s) k \frac{\hat{O}(s)}{\mu}-\frac{\left(\delta^{\prime}(s)\right)^{\alpha+1}}{(\alpha+1)^{\alpha+1}\left(\sigma^{\prime}(s)\right)^{\alpha} \pi^{\alpha}(s) \delta^{\alpha}(s)}\right) \mathrm{d} s \leq \frac{\delta(t)}{\pi^{\alpha} \sigma^{\alpha}(t)} .
$$

The proof is complete.

Lemma 9. Let $x(t)$ be a positive solution to (1) and $y(t)$, satisfying case $\mathbf{N}_{3}$. If

$$
\int_{t_{0}}^{\infty} \hat{O}(s) \mathrm{d} s=\infty
$$

or

$$
\int_{t_{0}}^{\infty} \frac{1}{r(s)}\left(\int_{t}^{\infty} \hat{O}(u) \mathrm{d} u\right)^{\frac{1}{\alpha}} \mathrm{d} s=\infty
$$

then $\lim _{t \rightarrow \infty} y(t)=0$.

Proof. Assume that $x(t)$ is a positive solution of (1) on $\left[t_{0}, \infty\right)$, there exists $t_{1} \geq t_{0}$ such that $x(\tau(t))>0$ and $x(\sigma(t))>0$ for all $t \geq t_{1}$. Since $y(t)>0$ and $y^{\prime}(t)<0$, there is $\lambda \geq 0$, such that $\lim _{t \rightarrow \infty} y(t)=\lambda$. Assume that $\lambda>0$. Integrating (6) from $t_{2}$ to $t$, we have

$$
\begin{aligned}
r(t)\left(y^{\prime \prime}(t)\right)^{\alpha}+\frac{p_{0}^{\alpha}}{\tau_{0}} r\left(y^{\prime \prime}(\tau(t))\right)^{\alpha} \leq & r\left(t_{1}\right)\left(y^{\prime \prime}\left(t_{1}\right)\right)^{\alpha}+\frac{p_{0}^{\alpha}}{\tau_{0}} r\left(\tau\left(t_{1}\right)\right)\left(y^{\prime \prime}\left(\tau\left(t_{1}\right)\right)\right)^{\alpha} \\
& -\frac{k}{\mu} \int_{t_{2}}^{t} \hat{O}(s) y^{\alpha}(\sigma(s)) \mathrm{d} s \\
\leq & r\left(t_{1}\right)\left(y^{\prime \prime}\left(t_{1}\right)\right)^{\alpha}+\frac{p_{0}^{\alpha}}{\tau_{0}} r\left(\tau\left(t_{1}\right)\right)\left(y^{\prime \prime}\left(\tau\left(t_{1}\right)\right)\right)^{\alpha} \\
& -\frac{k}{\mu} \lambda^{\alpha} \int_{t_{2}}^{t} \hat{O}(s) \mathrm{d} s .
\end{aligned}
$$

This contradicts (35). Hence $\lambda=0$. The proof is complete.

Theorem 6. Let $x(t)$ be a positive solution of (1). If

$$
\limsup _{t \rightarrow \infty} \int_{\sigma(t)}^{t} \hat{O}(s) R(\sigma(t), \sigma(s)) \mathrm{d} s>\frac{\tau_{0}}{\tau_{0}+p_{0}^{\alpha}},
$$

then case $\mathbf{N}_{3}$ is impossible, where $R(v, u)=\int_{u}^{v} \int_{\zeta}^{v} \frac{1}{r^{\frac{1}{\alpha}}(\zeta)} \mathrm{d} \zeta$.

Proof. Since $r\left(y^{\prime \prime}(t)\right)^{\alpha}$ is nonincreasing, pick $t_{1} \in\left[t_{0}, \infty\right)$ for $t \geq t_{1}$, we see that

$$
-y^{\prime}(u) \geq \int_{u}^{v} \frac{1}{r^{\frac{1}{\alpha}}(s)} r^{\frac{1}{\alpha}}(s) y^{\prime \prime}(s) \mathrm{d} s \geq r^{\frac{1}{\alpha}}(v) y^{\prime \prime}(v) \int_{u}^{v} \frac{1}{r^{\frac{1}{\alpha}}(s)} \mathrm{d} s,
$$


for $v \geq u$. Integrating above inequality from $u$ to $v$, we have

$$
y(u) \geq r^{\frac{1}{\alpha}}(v) y^{\prime \prime}(v) \int_{u}^{v} \int_{\zeta}^{v} \frac{1}{r^{\frac{1}{\alpha}}(\zeta)} \mathrm{d} \zeta=r^{\frac{1}{\alpha}}(v) y^{\prime \prime}(v) R(v, u) .
$$

Integrating (6) from $\sigma(t)$ to $t$ and using (38), we get (17)

$$
\begin{aligned}
\left(r(\sigma(t))\left(y^{\prime \prime}(\sigma(t))\right)^{\alpha}+\frac{p_{0}^{\alpha}}{\tau_{0}} r(\tau(\sigma(t)))\left(y^{\prime \prime}(\tau(\sigma(t)))^{\alpha}\right)\right) & \geq \frac{k}{\mu} \int_{\sigma(t)}^{t} \hat{O}(s) y^{\alpha}(\sigma(s)) \mathrm{d} s \\
& \geq \frac{k}{\mu} r(\sigma(t))\left(y^{\prime \prime}(\sigma(t))\right)^{\alpha} \int_{\sigma(t)}^{t} \hat{O}(s) R^{\alpha}(\sigma(t), \sigma(s)) \mathrm{d} s .
\end{aligned}
$$

Using the fact that $\left(r(t)\left(y^{\prime \prime}(t)\right)^{\alpha}\right)^{\prime}<0$ and (17), we obtain

$$
r(\sigma(t))\left(y^{\prime \prime}(\sigma(t))\right)^{\alpha}<r(\tau(\sigma(t)))\left(y^{\prime \prime}(\tau(\sigma(t)))\right)^{\alpha},
$$

and

$$
\begin{aligned}
r(\tau(\sigma(t)))\left(y^{\prime \prime}(\tau(\sigma(t)))^{\alpha}\right)\left(1+\frac{p_{0}^{\alpha}}{\tau_{0}}\right) & \geq-\frac{k}{\mu} r(\sigma(t))\left(y^{\prime \prime}(\sigma(t))\right)^{\alpha} \int_{\sigma(t)}^{t} \hat{O}(s) R^{\alpha}(\sigma(t), \sigma(s)) \mathrm{d} s \\
& \geq-\frac{k}{\mu} r(\tau(\sigma(t)))\left(y^{\prime \prime}(\tau(\sigma(t)))\right)^{\alpha} \int_{\sigma(t)}^{t} \hat{O}(s) R^{\alpha}(\sigma(t), \sigma(s)) \mathrm{d} s .
\end{aligned}
$$

Hence,

$$
\left(1+\frac{p_{0}^{\alpha}}{\tau_{0}}\right) \geq-\frac{k}{\mu} \int_{\sigma(t)}^{t} \hat{O}(s) R^{\alpha}(\sigma(t), \sigma(s)) \mathrm{d} s .
$$

This led to a contradiction. The proof is complete.

\section{Applications}

\subsection{Asymptotic Properties}

By combining Theorems 2-5 with Lemma 9, one can easily provide new criteria for the asymptotic properties of (1) as follows

Theorem 7. Assume that (13) holds. Then, (1) is almost oscillatory .

Theorem 8. Assume that (19) and (35) or (36) hold. Then (1) is almost oscillatory .

Theorem 9. Assume that (11), (22) and either (35) or (36) hold. Then, (1) is almost oscillatory.

Theorem 10. Assume that (11) holds and, if there is a nondecreasing function $\rho \in C^{1}\left(\left[t_{0}, \infty\right),(0, \infty)\right)$ and $\sigma^{\prime}(t)>0$, such that (28) and either (35) or (36) hold, then (1) is almost oscillatory.

Theorem 11. Assume that (11) holds and if there is a nondecreasing function $\delta \in C^{1}\left(\left[t_{0}, \infty\right),(0, \infty)\right)$, such that (29) and either (35) or (36) hold, then (1) is almost oscillatory.

\subsection{Oscillation}

In the following Theorem, we combine Theorems 2-5 with Theorem (37) to obtain new criteria for oscillation of (1)

Theorem 12. If all assumptions of Theorem 1 or 2 or 3 or 4 or 5 and (37) hold, then (1) is oscillatory.

Remark 2. Compared to the existing results of $[25,26]$, oscillation of (1) is attained by easier conditions. 
Example 1. Consider the third-order neutral delay differential equation

$$
\left(t^{2}\left(x(t)+p_{0} x(\epsilon t)\right)^{\prime \prime}\right)^{\prime}+\frac{q_{0}}{t} y(0.5 t)=0, t \geq 1
$$

where $\epsilon \in(0,1)$ and $q_{0}>0$. We note that $r=t^{2}, \sigma(t)=0.5 t, \tau(t)=\epsilon t, p(t)=p_{0}$. It can easily be verified that $\hat{O}(t)=\frac{q_{0}}{t}$. By choosing $\delta(t)=\pi(t) \tau(t)=\epsilon$, Condition (19), (29), (28) and (37) become

$$
\begin{aligned}
& q_{0}>\frac{2\left(\tau_{0}+p_{0}\right)}{\ln (2) \tau_{0} e} \\
& q_{0}>\frac{2}{\left(1-p_{0}\right)}, \\
& q_{0}>\frac{\left(\tau_{0}+p_{0}\right)}{2 \tau_{0}}
\end{aligned}
$$

and

$$
q_{0}>\frac{\tau_{0}}{\left(\tau_{0}+p_{0}\right)\left(0.5+\ln 0.5+\frac{1}{2} \ln ^{2} 0.5\right)}
$$

respectively. Using Theorems 8, 10 and 11, Equation (39) is almost oscillatory if (40) or (41) or (42) holds. Moreover, by Theorem 12, we see that (39) is oscillatory if

$$
q_{0}>\max \left\{\frac{\tau_{0}}{\left(\tau_{0}+p_{0}\right)\left(0.5+\ln 0.5+\frac{1}{2} \ln ^{2} 0.5\right)}, \frac{2\left(\tau_{0}+p_{0}\right)}{\ln (2) \tau_{0} e}\right\} .
$$

Remark 3. It is easy to verify that condition (13) fails; therefore, Theorem 1 does not apply.

Remark 4. If $p_{0}=1$ then our results are reduced to the results of Chatzarakis in [14].

Example 2. Consider the third-order neutral delay differential equation

$$
\left(t^{2}\left(\left(x(t)+p_{0} x(\epsilon t)\right)^{\prime \prime}\right)^{\alpha}\right)^{\prime}+\frac{q_{0}}{t} y^{\alpha}(\lambda t)=0, t \geq 1
$$

where $q_{0}>0$ and $\lambda, \epsilon \in(0,1)$. Condition (19), (28) and (37) reduse to

$$
\begin{aligned}
& C 1: q_{0}>\frac{\epsilon+p_{0}}{\lambda \epsilon e \ln \left(\frac{1}{\lambda}\right)^{\prime}} \\
& C 2: q_{0}>\frac{\mu\left(\epsilon+p_{0}^{\alpha}\right)^{1 / \alpha}}{4 \lambda \epsilon^{1 / \alpha}}
\end{aligned}
$$

and

$$
\mathrm{C} 3: q_{0}>\frac{\epsilon}{\left(\epsilon+p_{0}^{\alpha}\right)\left(1-\lambda+\ln \lambda+\frac{1}{2} \ln ^{2} \lambda\right)}
$$

respectively. Therefore, by Theorem 12, we see that (43) is oscillatory if

$$
q_{0}>\max \left\{\frac{\epsilon+p_{0}}{\lambda \epsilon e \ln \left(\frac{1}{\lambda}\right)}, \frac{\epsilon}{\left(\epsilon+p_{0}^{\alpha}\right)\left(1-\lambda+\ln \lambda+\frac{1}{2} \ln ^{2} \lambda\right)}\right\}
$$

or

$$
q_{0}>\max \left\{\frac{\mu\left(\epsilon+p_{0}^{\alpha}\right)^{1 / \alpha}}{4 \lambda \epsilon^{1 / \alpha}}, \frac{\epsilon}{\left(\epsilon+p_{0}^{\alpha}\right)\left(1-\lambda+\ln \lambda+\frac{1}{2} \ln ^{2} \lambda\right)}\right\} .
$$


Remark 5. Consider a particular case of (43), namely,

$$
\left(t^{2}\left(\left(x(t)+\frac{1}{4} x\left(\frac{t}{2}\right)\right)^{\prime \prime}\right)\right)^{\prime}+\frac{q_{0}}{t} y(\lambda t)=0,
$$

Conditions (44) and (45) reduce to

$$
q_{0}>\max \left\{\frac{\frac{3}{4}}{\frac{1}{4} \lambda e \ln \left(\frac{1}{\lambda}\right)}, \frac{\frac{1}{2}}{\left(\frac{3}{4}\right)\left(1-\lambda+\ln \lambda+\frac{1}{2} \ln ^{2} \lambda\right)}\right\}
$$

and

$$
q_{0}>\max \left\{\frac{3}{8 \lambda}, \frac{2}{3\left(1-\lambda+\ln \lambda+\frac{1}{2} \ln ^{2} \lambda\right)}\right\},
$$

respectively; see Figure 1. Thus, by Theorem 12, Equation (46) is oscillatory if (47) or (48) satisfies. So, For a given $\lambda \in(0,0.21)$, Condition (47) is sharp for oscillation, but in $\lambda \in(0.21,1)$ Condition (48) is sharp for oscillation.

On the other hand, consider a particular case of (43), namely,

$$
\left(t^{2}\left(\left(x(t)+\frac{1}{4} x\left(\frac{t}{2}\right)\right)^{\prime \prime}\right)^{\alpha}\right)^{\prime}+\frac{q_{0}}{t} y\left(\frac{t}{4}\right)=0
$$

where $\alpha>1$. Conditions (44) and (45) reduce to

$$
q_{0}>\max \left\{\frac{3}{e \ln 2}, \frac{1}{2} \frac{2^{2 \alpha+1}}{\left(2^{2 \alpha}+2\right)\left(2 \ln ^{2} 2-2 \ln 2+\frac{3}{4}\right)}\right\}
$$

or

$$
q_{0}>\max \left\{\frac{2}{17} 2^{\alpha}\left(\frac{1}{2^{2 \alpha}}\left(2^{2 \alpha}+2\right)\right)^{\frac{1}{\alpha}}, \frac{1}{2} \frac{2^{2 \alpha+1}}{\left(2^{2 \alpha}+2\right)\left(2 \ln ^{2} 2-2 \ln 2+\frac{3}{4}\right)}\right\}
$$

Remark 6. It is easy to notice that the effect of the delay argument on the oscillation parameters varies from one example to another, and no consistent pattern can be found to determine this effect. Additionally, the oscillation test depends on two different conditions, so we notice the change in the effect of the delay argument on oscillation (from inverse to direct relationship). This also applies to the effect of $\alpha$.

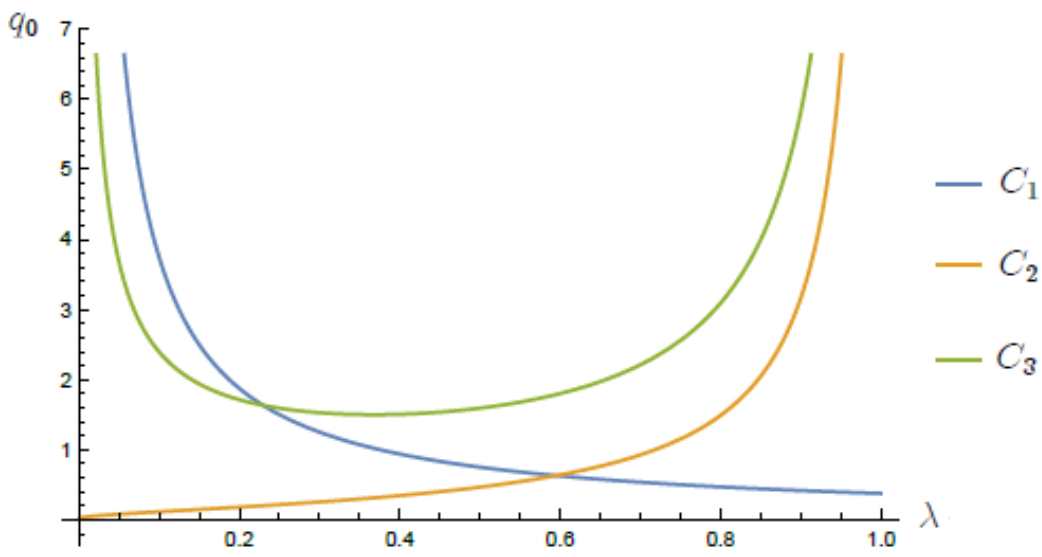

Figure 1. Test of the strength of criteria for (46). 


\section{Conclusions}

In this paper, we introduced a simplified theorem for near oscillation; furthermore, we established oscillation criteria for (1). Using comparison theorems and the Riccati technique, we established criteria to check the oscillation under fewer restrictions, and compared this with some results published in the literature. Our results are an extension of and complement to existing results in some previous studies, such as $[15,27,29]$.

The establishment of criteria for the oscillation of Equation (1) without the need for a condition $\sigma \circ \tau=\tau \circ \sigma$ and $\tau^{\prime}(t) \geq \tau_{0}$ remains an open problem.

Author Contributions: Conceptualization, B.Q., O.M., S.S.S., S.N., D.S. and E.M.E.; methodology, B.Q., O.M., S.S.S., S.N., D.S. and E.M.E.; validation, B.Q., O.M., S.S.S., S.N., D.S. and E.M.E.; formal analysis, B.Q., O.M., S.S.S., S.N., D.S. and E.M.E.; investigation,B.Q., O.M., S.S.S., S.N., D.S. and E.M.E.; writing-review and editing, B.Q., O.M., S.S.S., S.N., D.S. and E.M.E.; supervision, B.Q., O.M., S.S.S., S.N., D.S. and E.M.E.; funding acquisition, D.S.; All authors have read and agreed to the published version of the manuscript.

Funding: D.S. was funded out under State Assignment Projects (No. FWEU-2021-0006, FWEU-20210001) of the Fundamental Research Program of Russian Federation 2021-2030 using the resources of the High-Temperature Circuit Multi-Access Research Center (Ministry of Science and Higher Education of the Russian Federation, project no 13.CKP.21.0038).

Conflicts of Interest: There are no competing interests.

\section{References}

1. Liu, M.; Dassios, I.; Tzounas, G.; Milano, F. Stability Analysis of Power Systems with Inclusion of Realistic-Modeling of WAMS Delays. IEEE Trans. Power Syst. 2019, 34, 627-636. [CrossRef]

2. Milano, F.; Dassios, I. Small-Signal Stability Analysis for Non-Index 1 Hessenberg Form Systems of Delay Differential-Algebraic Equations. IEEE Trans. Circuits Syst. Regul. Pap. 2016, 63, 1521-1530. [CrossRef]

3. Agarwal, R.P.; Berezansky, L.; Braverman, E.; Domoshnitsky, A. Nonoscillation Theory of Functional Differential Equations with Applications; Springer: Berlin/Heidelberg, Germany, 2012; 520p, ISBN978-1-4614-3454-2.

4. Hale, J.K. Theory of Functional Differential Equations; Springer: Berlin/Heidelberg, Germany, 1977.

5. Moaaz, O.; Elabbasy, E.M.; Qaraad, B. An improved approach for studying oscillation of generalized Emden-Fowler neutral differential equation. J. Inequal. Appl. 2020, 2020, 69. [CrossRef]

6. Chatzarakis, G.E.; Moaaz, O.; Li, T.; Qaraad, B. Some oscillation theorems for nonlinear second-order differential equations with an advanced argument. Adv. Differ. Equ. 2020, 160. [CrossRef]

7. Bohner, M.; Grace, S.R.; Sager, Tunc, E. Oscillation of third-order nonlinear damped delay differential equations. Appl. Math. Comput. 2016, 278, 21-32. [CrossRef]

8. Chatzarakis, G.E.; Grace, S.R.; Jadlovska, I.; Li, T.; Tunc, E. Oscillation criteria for third-order Emden-Fowler differential equations with unbounded neutral coefficients. Complexity 2019, 2019, 5691758. [CrossRef]

9. Grace, S.R.; Graef, J.R.; Tunc, E. Oscillatory behaviour of third order nonlinear differential equations with a nonlinear nonpositive neutral term. J. Taibah Univ. Sci. 2019, 13, 704-710. [CrossRef]

10. Grace, S.R.; Jadlovska, I.; Tunc, E. Oscillatory and asymptotic behavior of third-order nonlinear differential equations with a superlinear neutral term. Turk. J. Math. 2020, 44, 1317-1329. [CrossRef]

11. Moaaz, O.; Qaraad, B.; El-Nabulsi, R.; Bazighifan, O. New Results for Kneser Solutions of Third-Order Nonlinear Neutral Differential Equations. Mathematics 2020, 8, 686. [CrossRef]

12. Elabbasy, E.M.; Qaraad, B.; Abdeljawad, T.; Moaaz, O. Oscillation Criteria for a Class of Third-Order Damped Neutral Differential Equations. Symmetry 1988, 12, 2020 [CrossRef]

13. Philos, C. On the existence of nonoscillatory solutions tending to zero at $\infty$ for differential equations with positive delays. Arch. Math. 1981, 36, 168-178. [CrossRef]

14. Chatzarakis, G.E.; Dzurina, J.; Jadlovsk, I. Oscillatory and asymptotic properties of third-order quasilinear delay differential equations. J. Inequal. Appl. 2019, 23. [CrossRef]

15. Li, T.; Zhang, C.; Baculikova, B.; Dzurina, J. On the oscillation of third-order quasi-linear delay differential equations. Tatra Mt. Math. Publ. 2011, 48, 117-123. [CrossRef]

16. Dzurina, J.; Thapani, E.; Tamilvanan, S. Oscillation of solutions to third-order half-linear neutral differential equations. Electron. J. Differ. Equ. 2012, 29, 1-9.

17. Graef, J.; Tunc, E.; Grace, S. Oscillatory and asymptotic behavior of a third-order nonlinear neutral differential equation. Opuscula Math. 2017, 37, 839-852. [CrossRef]

18. Santra, S.S.; Ghosh, A.; Bazighifan, O.; Khedher, K.M.; Nofal, T.A. Second-order impulsive differential systems with mixed and several delays. Adv. Differ. Equ. 2021, 1, 1-12. [CrossRef] 
19. Santra, S.S.; Baleanu, D.; Khedher, K.M.; Moaaz, O. First-order impulsive differential systems: Sufficient and necessary conditions for oscillatory or asymptotic behavior. Adv. Differ. Equ. 2021, 1, 1-20. [CrossRef]

20. Santra, S.S.; Tripathy, A.K. On oscillatory first order nonlinear neutral differential equations with nonlinear impulses. J. Appl. Math. Comput. 2019, 59, 257-270. [CrossRef]

21. Ruggieri, M.; Santra, S.S.; Scapellato, A. On nonlinear impulsive differential systems with canonical and non-canonical operators Appl. Anal. 2021. [CrossRef]

22. Moaaz, O. Oscillation Theorems for Cartain Second-Order Differential Equations; Lambert Academic Publishing: Saarbrücken, Germany, 2014.

23. Tunc, E. Oscillatory and asymptotic behavior of third-order neutral differential equations with distributed deviating arguments. Electron. J. Differ. Equ. 2017. [CrossRef]

24. Tiryaki, A.; Aktas, M.F. Oscillation criteria of a certain class of third-order nonlinear delay differential equations with damping. J. Math. Anal. Appl. 2007, 325, 54-68. [CrossRef]

25. Baculikova, B.; Dzurina, J. Oscillation of third-order functional differential equations. Electron. J. Qual. Theory of Diff. Equ. 2010, 43, 1-10. [CrossRef]

26. Baculikova, B.; Dzurina, J. Oscillation of third-order nonlinear differential equations. Appl. Math. Lett. 2011, 24, 466-470. [CrossRef]

27. Grace, S.R.; Agarwal, R.P.; Pavani, R.; Thapani, E. On the oscillation of certain third order nonlinear functional differential equations. Appl. Math. Comput. 2008, 202, 102-112. [CrossRef]

28. Saker, S.H.; Dzurina, J. On the oscillation of certain class of third-order nonlinear delay differential equations. Math. Bohem. 2010, 135, 225-237. [CrossRef]

29. Ravi, P.; Agarwal, R.P.; Bohner, M.; Li, T.; Zhang, C. Oscillation of Third-Order Nonlinear Delay Differential Equations. Taiwan. J. Math. 2013, 17, 545-558.

30. Sidorov, N.A.; Trufanov, A.N. Nonlinear operator equations with a functional perturbation of the argument of neutral type. Differ. Equ. 2009, 45, 1840-1844. [CrossRef]

31. Zhang, S.Y.; Wang, Q. Oscillation of Second-Order Nonlinear Neutral Dynamic Equations on Time Scales. Appl. Math. Comput. 2010, 216, 2837-2848. [CrossRef]

32. Thapani, E.; Li, T. On the oscillation of third-order quasi-linear neutral functional differential equations. Arch. Math. 2011, 47, 181-199. 